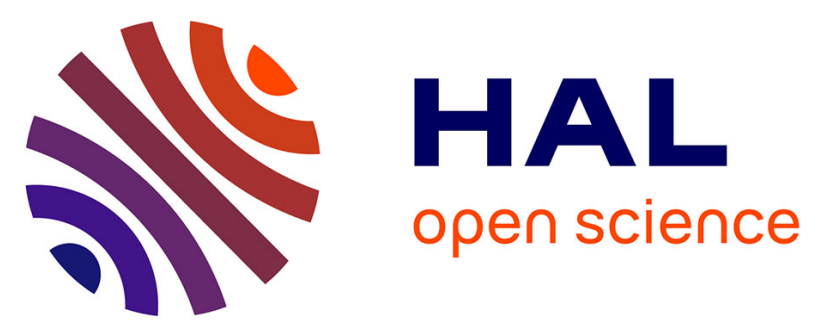

\title{
Assessing the influence of real releases on explosions : selected results from large-scale experiments
}

Helene Hisken, Lorenzo Mauri, Gordon Atanga, Melodia Lucas, Kees van Wingerden, Trygve Skjold, Pierre Quillatre, Antoine Dutertre, Thibault Marteau, Andrzej Pekalski, et al.

\section{To cite this version:}

Helene Hisken, Lorenzo Mauri, Gordon Atanga, Melodia Lucas, Kees van Wingerden, et al.. Assessing the influence of real releases on explosions: selected results from large-scale experiments. 13th International symposium on hazards, prevention, and mitigation of industrial explosions (ISHPMIE), Jul 2020, Braunschweig, Germany. ineris-03319940

\section{HAL Id: ineris-03319940 \\ https://hal-ineris.archives-ouvertes.fr/ineris-03319940}

Submitted on 13 Aug 2021

HAL is a multi-disciplinary open access archive for the deposit and dissemination of scientific research documents, whether they are published or not. The documents may come from teaching and research institutions in France or abroad, or from public or private research centers.
L'archive ouverte pluridisciplinaire HAL, est destinée au dépôt et à la diffusion de documents scientifiques de niveau recherche, publiés ou non, émanant des établissements d'enseignement et de recherche français ou étrangers, des laboratoires publics ou privés. 


\title{
Assessing the influence of real releases on explosions: selected results from large-scale experiments
}

\author{
Helene Hisken ${ }^{a}$, Lorenzo Mauri ${ }^{a}$, Gordon Atanga ${ }^{a}$, Melodia Lucas ${ }^{a}$, Kees van Wingerden ${ }^{a}$, \\ Trygve Skjold $^{b}$, Pierre Quillatre ${ }^{c}$, Antoine Dutertre ${ }^{d}$, Thibault Marteau ${ }^{d}$, Andrzej Pekalski ${ }^{e}$, \\ Lorraine Jenney $^{f}$, Dan Allason ${ }^{f}$, Mike Johnson ${ }^{f}$, Emmanuel Leprette ${ }^{g}$, Didier Jamois ${ }^{g}$, \\ Jérôme Hébrard ${ }^{g}$ \& Christophe Proust ${ }^{g}$ \\ ${ }^{a}$ Gexcon R\&D, Bergen, Norway \\ ${ }^{b}$ University of Bergen, Norway \\ ${ }^{c}$ GRTgaz, Research and Innovation Center for Energy, France \\ ${ }^{d}$ Total, France \\ ${ }^{e}$ Shell Global Solutions, UK \\ ${ }^{f}$ DNV GL Spadeadam Research and Testing, UK \\ ${ }^{g}$ Ineris, Verneuil-en-Halatte, France
}

E-mail: Helene.Hisken@gexcon.com

\begin{abstract}
The research activities in the project Assessing the Influence of Real Releases on Explosions (AIRRE) included a unique series of large-scale explosion experiments with ignited high-momentum jet releases directed into congested geometries. The primary objective for the AIRRE project was to gain improved understanding of the effect realistic releases and turbulent flow conditions have on the consequences of accidental gas explosions in the petroleum industry, to develop methodology that can facilitate safe and optimal design of process facilities. This paper presents selected results from experiments involving ignition of a highly turbulent gas cloud, generated by a large-scale, pressurised release of natural gas. The paper gives an overview of the effect on maximum explosion overpressures of varying the ignition position relative to the release point of the jet and a congested region placed inside the flammable cloud, with either a high or medium level of congestion. For two of the tests involving a jet release and the medium congestion rig, the maximum overpressures significantly exceeded those obtained in a quiescent reference test. The paper presents detailed results for selected tests in the campaign. The effects on the relevant explosion phenomena from introducing initial turbulence, initial flow momentum and concentration gradients, as generated by the realistic release, are discussed.
\end{abstract}

Keywords: realistic releases, large-scale experiments, gas explosions

\section{Introduction}

According to Marsh (2018), a majority of the 100 largest property losses in the hydrocarbon industries from 1974 to 2017 involved fires and explosions. In order to assess the potential consequences of such events, and implement effective risk-reducing measures, the industry requires knowledge about the underlying physical phenomena. Accidental gas explosions will typically include loss of containment of gaseous or liquid fuel, dispersion, mixing to form a flammable fuel-air cloud, ignition, turbulent combustion, pressure build-up, and propagation of blast waves in the surroundings (CCPS, 1994). Furthermore, recent incidents such as the vapour cloud explosions in Jaipur in 2009 and Skikda in 2004, as well as the extensive research conducted after the Buncefield incident in 2005, highlight the importance of considering deflagration-to-detonation transition (DDT) in risk assessments (Johnson et al. 2015; Oran et al., 2020). 
Gas explosions are inherently complex, as they involve physical phenomena on a wide range of spatial and temporal scales. The pressure loads from accidental gas explosions depend strongly on the rate of combustion, which is determined by factors such as degree of congestion, degree of confinement, the reactivity of the fuel-air mixture, and properties of the reactive flow. The primary mechanism for flame acceleration in congested geometries is the positive feedback between expansion of combustion products, turbulence generated in the unreacted mixture, especially in shear and boundary layers from flow past obstacles and walls, and enhanced combustion rates (Schelkin, 1940; Moen et al., 1980; Hjertager, 1984; Bjerketvedt et al., 1997). In a flammable fuel-air cloud generated by a high-pressure release, a certain degree of pre-ignition turbulence will normally be present. Initial turbulence can significantly enhance the rate of flame acceleration in gas explosions (Skjold et al., 2018). Furthermore, if ignition happens while the release is still on-going, the initial mean flow speed can be relatively high. This affects how the flame front propagates in different regions (with or against the flow) as well as the properties of the wakes generated downstream of obstructions prior to ignition. Significant concentration gradients may be present in the cloud, and regions of reduced reactivity of the flammable mixture can produce oscillatory pressure pulses as the flame repeatedly speeds up and slows down (Johnson et al., 2015). Finally, it is likely that pre-ignition turbulence will reduce the runup distance to DDT (Davis et al., 2016; Davis et al., 2018), and hence increase the likelihood of detonation of a flammable cloud generated by a high-pressure release. It may not be straightforward to assess the combined effect of these factors.

Skjold et al. (2018) provided a detailed review of previous experimental work on the effect of realistic releases on explosions from the open literature. For example, as part of the project Blast and Fire Engineering for Topside Structures (BFETS), a range of gas explosion experiments were performed in full-scale or medium-scale offshore modules (Johnson \& Cleaver, 2002; Johnson et al., 2002). The campaigns included tests with gas clouds resulting from realistic, high-pressure releases of natural gas. In most tests, the releases generated clouds that were less reactive and/or had a less "optimal" distribution (with respect to explosion pressure generation after ignition) throughout the rigs than near-stoichiometric, homogeneous clouds. However, a few of the realistic release tests produced overpressures that were comparable to or even locally exceeded the pressures recorded in the corresponding reference experiments with homogeneous and quiescent clouds.

Probabilistic explosion studies frequently apply computational fluid dynamics (CFD) simulations where non-homogenous fuel-air clouds, generated by pressurised gaseous releases, are represented by so-called equivalent clouds (Hansen et al., 2013; Skjold et al., 2018; Stewart \& Gant, 2019). By replacing inhomogeneous and turbulent gas clouds with equivalent homogeneous fuel-air mixtures, that can be moved around and ignited in various positions, risk analysts can investigate numerous explosion scenarios (with comparatively short simulations) based on a relatively limited number of time-consuming release and dispersion simulations. Stewart \& Gant (2019) reviewed previous work and the scientific basis for the equivalent cloud concept. GDF SUEZ (currently GRTgaz) compared explosion pressures predicted with FLACS v10.2 in geometries with a medium or high level of congestion for scenarios involving jet releases of methane at flow rates of 1,10 or $100 \mathrm{~kg} / \mathrm{s}$ and the corresponding homogeneous and quiescent clouds defined according to the Q9 equivalent-cloud concept (Skjold et al., 2018). When applying the Q9 approach for the scenarios with a medium level of congestion, the maximum overpressures were approximately a factor two lower than the maximum overpressures obtained when igniting the clouds from the realistic releases.

Motivated by these results, the goal of the project Assessing the Influence of Real Releases on Explosions (AIRRE) was to gain improved understanding of the effect realistic releases and turbulent flow conditions have on the consequences of accidental gas explosions in the petroleum industry. From the open literature, there is limited data available that show the combined effects on gas explosion severity from pre-ignition turbulence generated by jet releases and explosion-generation turbulence. Furthermore, the spatial scales and flow rates for which previous experiments with delayed ignition of pressurised releases have been performed, are rather limited (Skjold et al., 2018). The AIRRE project included an experimental campaign with delayed ignition of natural gas jets with 
a mass flow in excess of $100 \mathrm{~kg} / \mathrm{s}$, either directed into congested regions with two different congestion levels or into an open area. The present paper discusses selected results from this campaign.

\section{Experiments}

\subsection{Experimental setup}

DNV GL Spadeadam Research and Testing conducted the large-scale experimental campaign in AIRRE at the Spadeadam test site in the UK. As part of the campaign, transient releases of natural gas were directed into obstructed regions that were placed at various distances from the release. The ignition point was varied relative to the position of the congested regions and the point of release.

The releases in the AIRRE project were fed from a storage reservoir containing approximately 22 metric tonnes of inventory when charged to its operating pressure of $150 \mathrm{barg}$ with natural gas. The storage reservoir is in a nominally flat area of the site measuring approximately $70 \mathrm{~m}$ wide by $320 \mathrm{~m}$ long. Fig. 1 shows an aerial view of the test site, with the release (blue) directed into two congested regions (red squares).

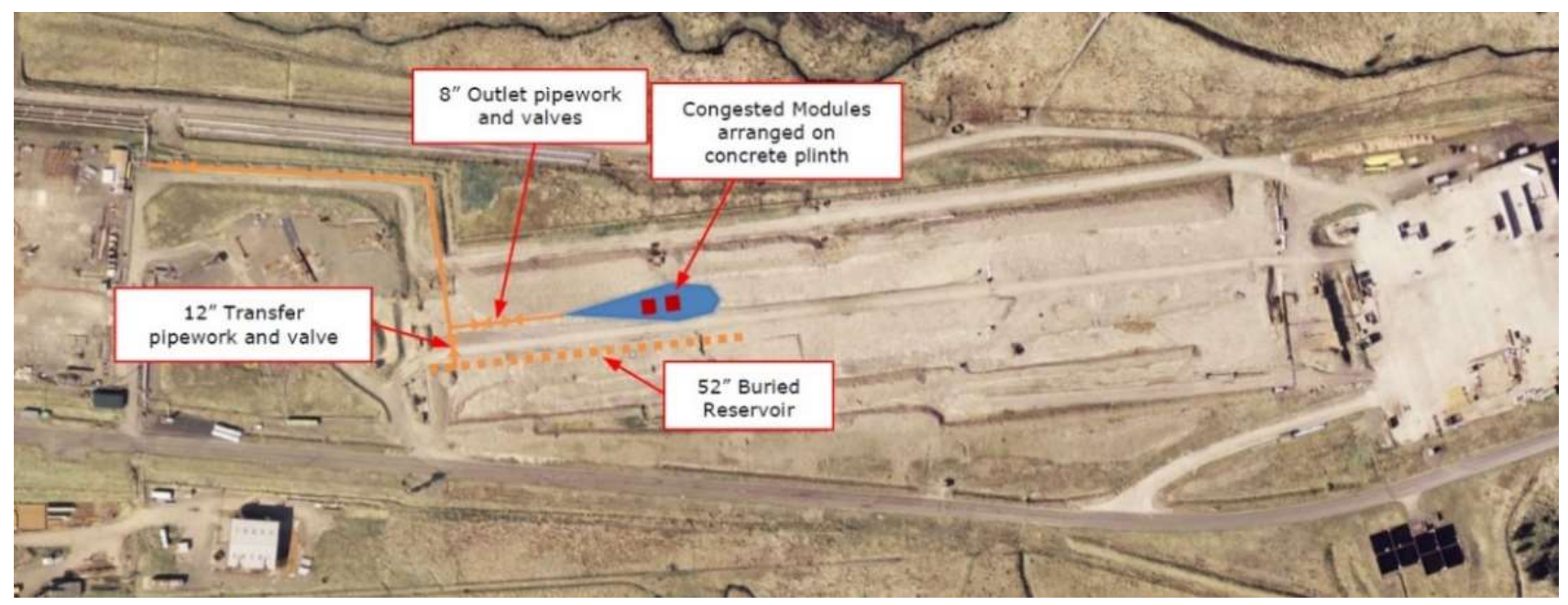

Fig. 1. Overview of test area.

The storage reservoir was connected to the $100 \mathrm{~mm}$ nozzle at the release point by a pipeline with initial diameter $0.3 \mathrm{~m}$ (12-inch), later reduced to $0.2 \mathrm{~m}$ (8-inch). The flow rate was calculated from the differential pressure over an orifice plate that was installed in the 8-inch pipework. In each test, a transient flow was driven by a reservoir pressure starting at approximately 132 barg and reducing to $70 \mathrm{barg}$, and ignition was initiated when the outlet pressure was around $75 \mathrm{barg}$. Using outlet pressure for timing ignition provided repeatable mass release rates that could be checked by the independent flow measurement. Ignition was accomplished by one of a set of three chemical fuses located in various positions. A backup igniter consisting of an incendiary firework was also installed, to be used if none of the other fuses were able to ignite the cloud. Shutdown of the release was initiated as soon as ignition occurred, however the actuated release valve took around 16 seconds to close, so a jet fire continued through this period.

Two different obstructed regions were used in the AIRRE project, one with a high level of congestion (denoted as "rig 7"), and one with a medium level of congestion (denoted as "rig 8"). Each obstructed region is constructed from pipes arranged in a three-dimensional mesh. Both rigs have dimensions of approximately $10.0 \mathrm{~m} \times 5.0 \mathrm{~m} \times 2.5 \mathrm{~m}$. The high congestion rig is made from 32 by 16 by 8 pipes with a diameter of approximately $76 \mathrm{~mm}$, while the medium congestion rig is made from 16 by 8 by 4 pipes with a diameter of approximately $168 \mathrm{~mm}$. The volume blockage of the high congestion rig is around $12 \%$, while the volume blockage for the medium congested rig is around $17 \%$. Fig. 2 illustrates the congestion level of the two rigs. 
One of the tests in the AIRRE project involved a homogeneous, nominally quiescent and nearstoichiometric natural gas-air cloud (i.e. not generated by a jet release). The flammable mixture was contained by a polythene sheet fastened to a $26 \mathrm{~m} \times 6.5 \mathrm{~m} \times 3 \mathrm{~m}$ steel angle frame. To achieve a homogenous gas concentration, a system of $600 \mathrm{~mm}$ diameter flap valves, a fan and polythene tubing was used to circulate the gas through the test rig. The medium congestion rig was placed inside the flammable cloud during the test. A spark igniter was installed within the test rig at ground level, positioned centrally at the edge of the congestion.
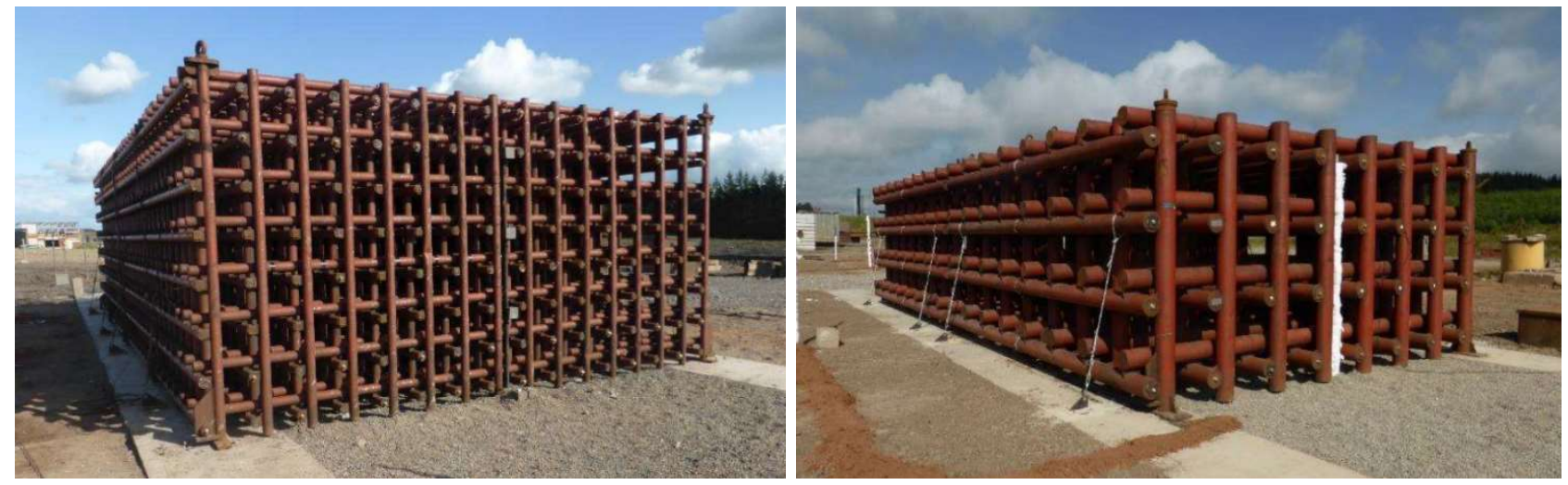

Fig. 2. The test rigs, with high (left) and medium (right) level of congestion, denoted as rig 7 and rig 8 , respectively.

The composition of the natural gas used in the campaign varied between 91-93 vol.\% methane, 5-7 vol.\% ethane, 0.6-0.7 vol.\% propane, 0.1-0.2 vol.\% I-butane, 0.1-0.2 vol.\% N-butane, and 0-0.4 vol.\% nitrogen.

\subsection{Instrumentation}

The pressure of the gas in the reservoir and pipework was monitored at four locations: one transducer was placed in the reservoir, one upstream of the metering orifice, one downstream of the metering orifice and one close to the exit orifice. The fluid temperature in the pipework was measured at three locations: one thermocouple in the reservoir, one in the pipe bore near to the metering orifice and one near to the release orifice. The mass flow rate of the gas through the orifice was determined using measurement of static wall fluid pressure, temperature and differential pressure across the orifice plate placed in the outlet pipework, following the method described in ISO 5167-1 (2003) and ISO 5167-2 (2003).

Sensors were installed to measure the fuel concentration, the explosion overpressure and the flame arrival time at several locations distributed throughout the gas plume in every test. The gas concentration in the plume was derived from measurements of oxygen depletion using City Technology AO2 (City Technology, 2017) (or equivalent) electro-chemical sensors. To minimise the effects of pressure and temperature fluctuations, the sensors were mounted in steel enclosures with a pressure-snubbing orifice. The concentration sensors were mounted on steel stands to enable measurement at different elevations from ground level. Section 3 includes further discussion on the concentration measurements.

Explosion overpressure was measured by a total of 20 PCB type M113B26 ICP pressure transducers (PCB Piezotronics, 2020), mounted at ground level in steel boxes. The faces of the pressure transducers were covered with a layer of silicone grease and silver foil in order to reduce thermal effects on the transducer. Measurement of flame arrival time was attempted by using an array of up to 28 ionisation probes. However, in the high-pressure jet release tests, the reliability of the sensors was limited due to the turbulent nature of the flow, the water vapour and the debris that was drawn up from the ground and into the jet release prior to ignition. Therefore, flame speed was instead estimated from high-speed video recordings of each experiment, as explained in Section 3. In one 
large-scale release test, thermal radiation after ignition was measured in 15 locations, using MEDTHERM ${ }^{\circledR}$ radiometers with a measuring range of $0-200 \mathrm{~kW} / \mathrm{m}^{2}$. The wind speed and direction were measured at 5 and $10 \mathrm{~m}$ above the local ground level, at a single location approximately $30 \mathrm{~m}$ behind the release point. The relative humidity was also measured $30 \mathrm{~m}$ behind the release point. Finally, in selected tests, up to 20 sensors were installed to measure turbulence in the flow prior to flame arrival (Jamois et al., 2020).

Signals from the dynamic pressure sensors, ionisation probes and turbulence sensors in each experiment were recorded at a sample frequency of $500 \mathrm{kHz}$. In jet release tests the gas concentration, pipework conditions, thermal radiation and ambient conditions were recorded at a rate of $10 \mathrm{~Hz}$. In the quiescent test, the gas concentration and rig conditions prior to ignition was recorded at a rate of $1 \mathrm{~Hz}$. Section 3 includes further details on the setups and measurements for a selection of the largescale tests performed as part of the AIRRE project.

\section{Results and discussion}

\subsection{Overview of results}

The AIRRE campaign included in total 16 tests: three tests with an un-obstructed jet, 8 tests with the medium congestion rig (including the quiescent reference test) and 5 tests with the high congestion rig. Fig. 3 shows the maximum overpressures that were measured in all 16 tests of the AIRRE campaign. The pressure-time traces used to obtain the maximum values in Fig. 3 have been filtered with a $0.1 \mathrm{~ms}$ moving average filter.

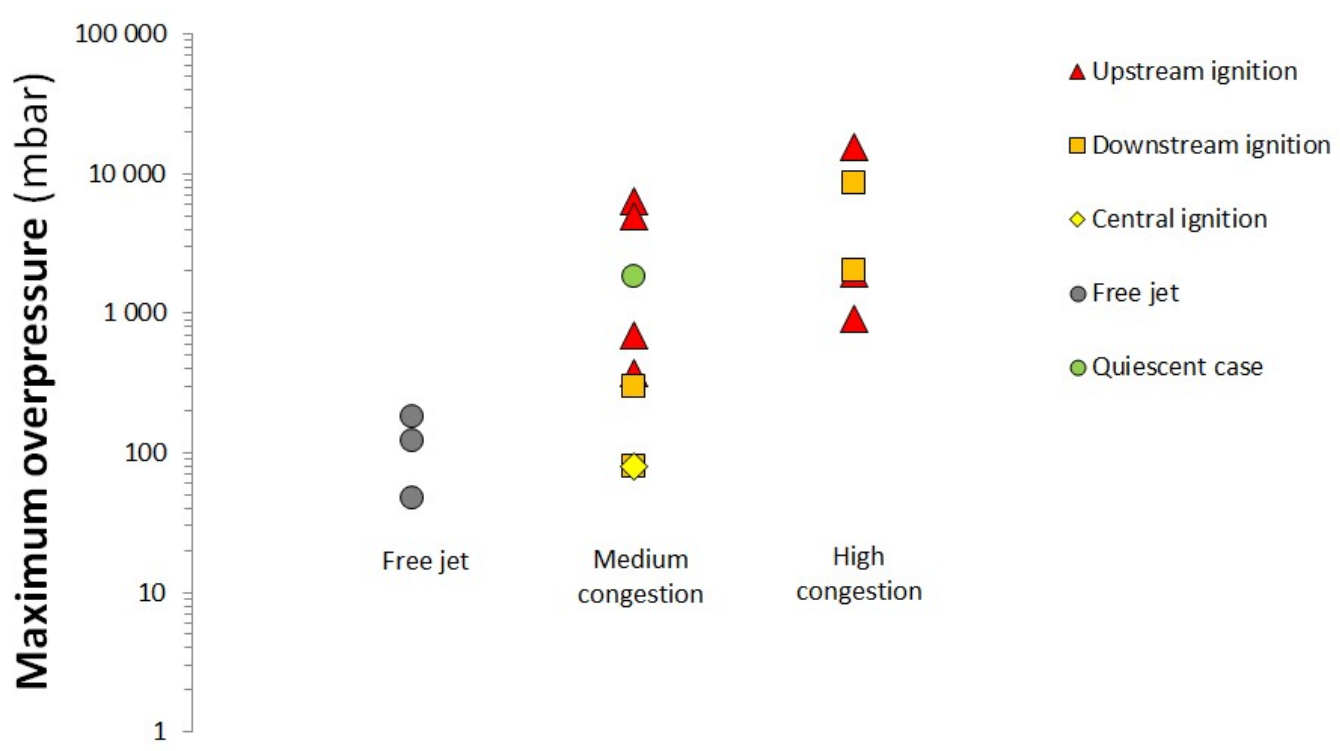

Fig. 3. Maximum explosion overpressures from all tests in the AIRRE campaign.

The significant spread in results for a given level of congestion and ignition annotation in Fig. 3 is partly caused by the fact that the ignition point relative to the release was varied between tests (for example at several different positions upstream of rig 8). Furthermore, in three of the tests with rig 8 , the congested region was placed at a distance from the point of release where the resulting fuel-air cloud was found to be fuel-rich. For all other tests, the congested regions were positioned $55 \mathrm{~m}$ from the release, where the average fuel-air concentration in the plume was expected to be nearstoichiometric. The presence of the jet release leads to inherent variability in the initial conditions at the time of ignition, due to fluctuations in the concentration and velocity fields.

Regardless of the variations in initial conditions between experiments, certain trends can be discerned from Fig. 3. The overall highest overpressures of the campaign were obtained with the high congestion rig in a test where ignition was initiated upstream of the congested region (the un-filtered 
peak value exceeded 30 bar). This maximum overpressure value is similar to overpressures obtained in a corresponding test with a homogeneous cloud from a previous campaign. Fig. 3 also shows that for the medium congestion level, the initial conditions generated by the realistic release can cause an explosion event that is significantly more severe than in the corresponding quiescent configuration. For example, this was the case for test 8 of the AIRRE campaign, which involved rig 8 positioned $55 \mathrm{~m}$ from the release point. Ignition was initiated upstream of the rig, $100 \mathrm{~mm}$ from ground level at the centre of the congested region's edge. The equivalent quiescent, near-stoichiometric natural gasair experiment, test 16, had the same ignition location relative to the congestion as in test 8 .

It may be reasonable to assume that ignition upstream of the congested region would enhance explosion severity compared to the tests with downstream ignition, as the flame front in this situation is pushed towards the obstructions by the mean flow. The flame speed and the shape of the flame front at entry into the congestion can have a significant effect on the explosion severity, cf. the observations by for example Cronin \& Wickens (1986), Tomlin \& Johnson (2013) and Davis in the RPSEA II project (S. Davis, private communication, 2019). This effect seems to be present in the tests with medium congestion in Fig. 3, while it is less pronounced for the high congestion rigs.

In the following, further results are presented for two of the tests performed as part of the AIRRE project: test 8 (medium congestion rig, ignition at the upstream edge of the congestion, $55 \mathrm{~m}$ from the point of release) and test $\mathbf{1 6}$ (reference test with a quiescent, homogeneous cloud enclosed in polythene tent, ignition at edge of congestion). Reference is also made to results from test 15 ("free" jet, i.e. without congested regions, ignition at $55 \mathrm{~m}$ from the release point).

\subsection{Concentration measurements}

In each of the large-scale jet release tests, pressurised natural gas was released horizontally, at approximately $1.4 \mathrm{~m}$ above ground level, from the $100 \mathrm{~mm}$ diameter nozzle. The release rate at ignition time varied between approximately 100 and $120 \mathrm{~kg} / \mathrm{s}$ between the different tests. Since the outflow of natural gas was not terminated immediately after ignition of the jet, a jet fire developed in the final stage of the experiment. Fig. 4 illustrates the different phases for one of the un-obstructed high-pressure release tests. Radiation measurements from one of the jet fires will be reported elsewhere.

Concentration sensors were placed along the release direction at $10 \mathrm{~m}$ intervals from $20 \mathrm{~m}$ to $80 \mathrm{~m}$ and at $100 \mathrm{~m}$, measured from the release point. The sensors were mounted $1.4 \mathrm{~m}$ above the ground on instrumentation stands. Additional sensors were positioned at selected distances from the release point (depending on each test), symmetrically around the centre line along the direction of the release, at $5 \mathrm{~m}$ and $10 \mathrm{~m}$ from the centre line. At certain distances, the concentration was measured at three different elevations, $0.7 \mathrm{~m}, 1.4 \mathrm{~m}$ and $2.1 \mathrm{~m}$ from the ground. In the tests with congested regions, a different layout of sensors was used in the rig area with positions relative to the rig. On the same stands, depending on the position, the instrumentation also included pressure transducers, turbulence sensors and ionisation probes. Fig. 5 shows the layout of the instrumentation for test 8, which had a medium congestion rig placed $55 \mathrm{~m}$ from the release point.

The concentration measurements were recorded by sensors that apply the oxygen-depletion principle, i.e. in the large-scale release experiments it is assumed that air is displaced by natural gas (cf. Section 2.2). The time response of the sensors to an oxygen concentration change from $20.9 \mathrm{vol} \%$ to $2.1 \mathrm{vol} \%$ is quoted by the manufacturer as being below 5 seconds. Since partial pressure diffusion is required to transfer oxygen to the sensing elements, the concentration sensors are sensitive to pressure and temperature fluctuations. In high-velocity flows, this effect results in a reduction in the apparent gas concentration, as the signal from the sensor is directly proportional to the pressure of the fluid. To evaluate the impact of such effects on the results, a set of small-scale commissioning tests were performed prior to the large-scale tests. Based on these results, it is assumed the dynamic pressure generated by flow velocities higher than $25 \mathrm{~m} / \mathrm{s}$ may underestimate the actual concentration. This can 
be seen in some of the concentration-time traces (measured close to the release point) as short term 'dips' in measured gas concentration.

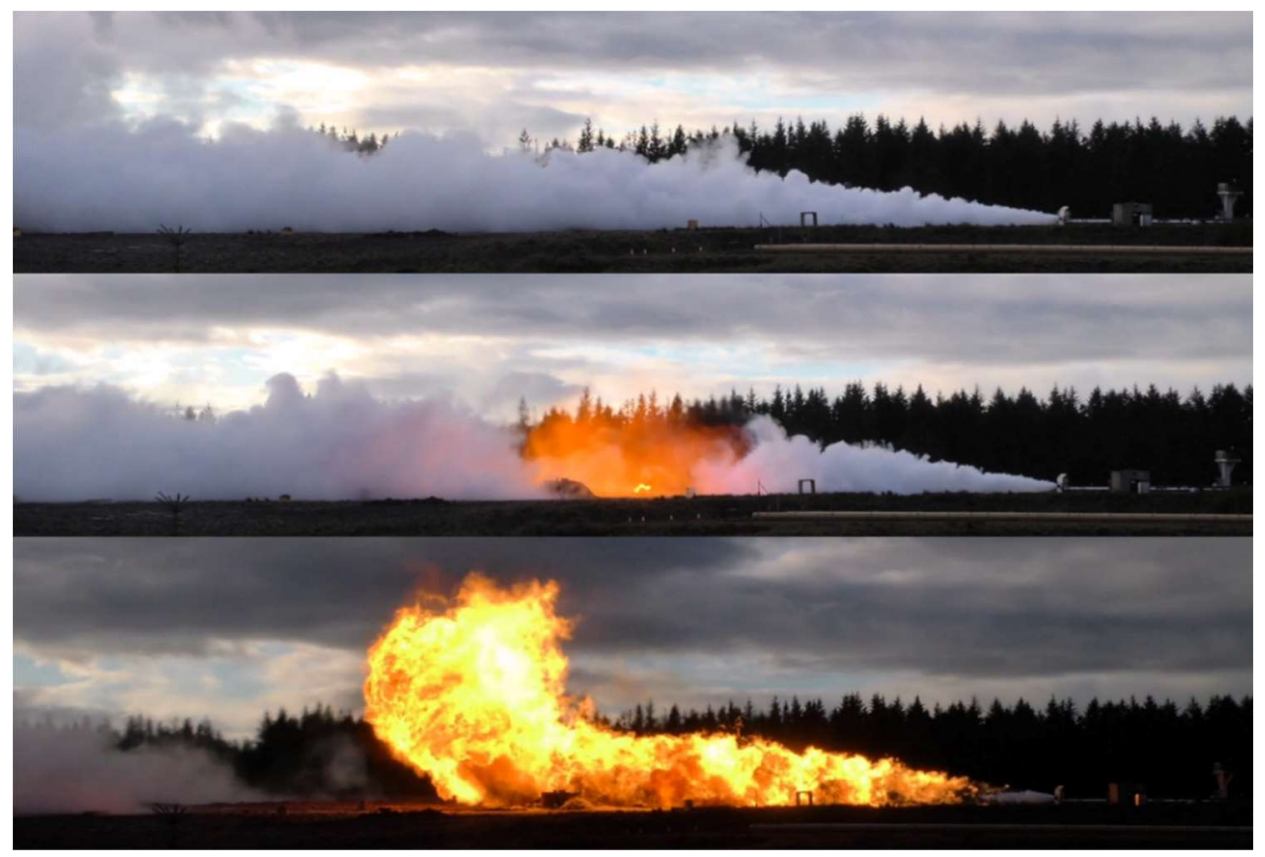

Fig. 4. Snapshots from different phases of a "free" jet release experiment: unignited jet release phase (top), flame propagation after ignition (middle) and jet fire phase (bottom).

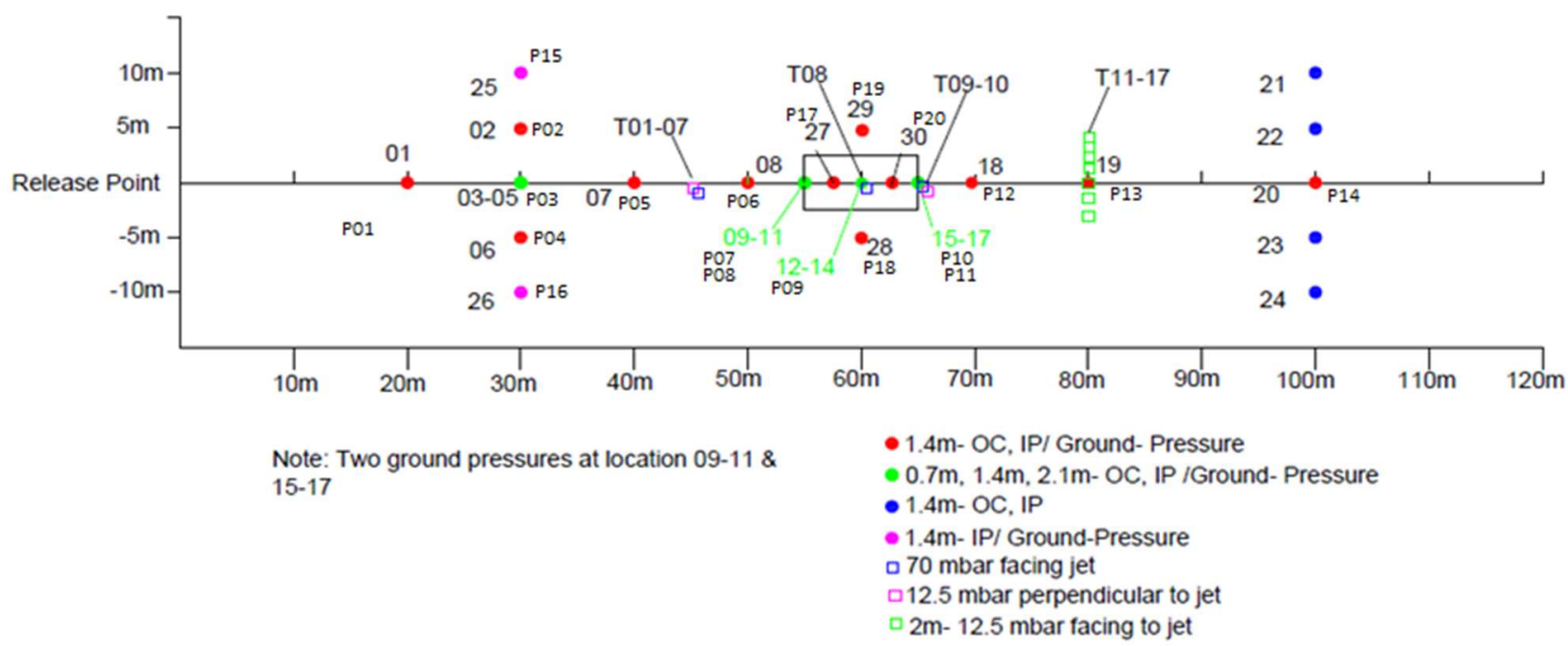

Fig. 5. Instrumentation layout for test 8 . The legend explains the plotting style for each sensor type (OC-Oxygen Cell, IP - Ionisation Probe, T- Turbulence Probe). The square markers denote different types and configurations of turbulence probes.

In the full-scale experiments, post-processing of turbulence measurements performed by Ineris (Jamois et al., 2020) showed that the jet centreline velocity at $40 \mathrm{~m}$ from the point of release was around $50 \mathrm{~m} / \mathrm{s}$, thus, concentration measurements at this distance and upstream may be significantly affected by dynamic pressure effects. In the following, concentration measurements upstream of 40 $\mathrm{m}$ from the release are therefore not included in the analysis. When interpreting the results for gas concentration at the time of ignition for the remaining sensors, the period maximum value recorded in a certain period before ignition is used. Inside the congested regions, post-processing of the turbulence measurements suggests that flow velocities were reduced to less than $20 \mathrm{~m} / \mathrm{s}$. The dynamic pressure effect on these sensors is therefore likely negligible. 
Fig. 6 shows the estimated mass flow rate across the orifice plate for tests 15 and 8 . The differential pressure signal, from which the rate is calculated, was aligned to zero at the time corresponding to the closure of the valve. The mass flow rates are similar from around $30 \mathrm{~s}$ prior to ignition - at this point the jet was likely quasi-steady. Minor variations in the initial pressure and temperature in the reservoir lead to variations of the mass flow rate of less than $+/-10 \%$ between tests.

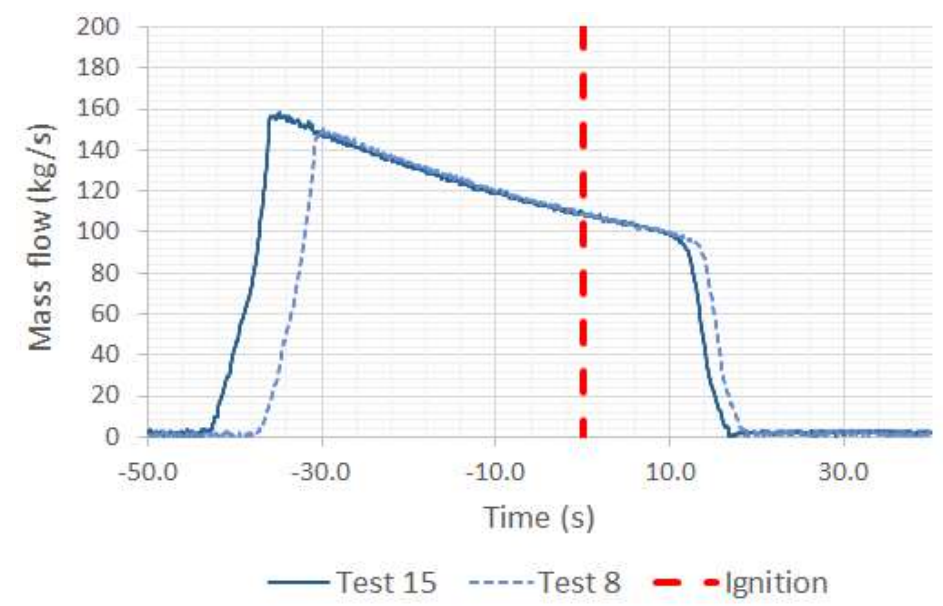

Fig. 6. Mass flow rate across orifice plate for tests 15 and 8 .

The wind speed and direction were measured before and during each test, at approximately $30 \mathrm{~m}$ behind the release point, at both 5 and $10 \mathrm{~m}$ above the local ground level. The average wind speed during the last $30 \mathrm{~s}$ before ignition, measured at an elevation of $5 \mathrm{~m}$ above the ground, varied between approximately 2 and $8 \mathrm{~m} / \mathrm{s}$ between tests. The effect of wind is likely limited close to the release and in the congested region but may affect the far-field concentrations where flow speeds are low. Humidity and condensation of water may have influenced the temperature of the plume. Similarly, atmospheric stability can change the relative buoyancy of the cloud and the background turbulence level. However, considering the high velocities generated by the jet, it is reasonable to assume that both humidity and atmospheric stability had a minor influence on concentrations above the lower flammability limit.

Fig. 7 shows the period maximum concentration (over $5 \mathrm{~s}$ before ignition) measured by selected sensors that were placed at $1.4 \mathrm{~m}$ above ground level in test 8 . Concentrations measured upstream of $40 \mathrm{~m}$ have been excluded, as already described, together with measurements from certain sensors that appeared to be erroneous. Similarly, Fig. 8 shows the maximum concentration measured (over a $5 \mathrm{~s}$ period before ignition) on the release centre line $(\mathrm{y}=0)$ at different elevations from ground level. The black rectangle marks the position of the rig in both Fig. 7 and Fig. 8.

Fig. 9 shows selected concentration measurements in time from sensors located $1.4 \mathrm{~m}$ from the ground level, along the centreline of the release, at various distances from the release orifice. The profile for the $50 \mathrm{~m}$ location show that there are still some effects due to the dynamic pressure from the jet on the measurements at this point; there are fewer oscillations and less noise on the traces that were recorded further away from the point of release and within the congested region. The increase in concentration at $62.5 \mathrm{~m}$ from the release was measured either slightly before or at the same time as that measured by the three sensors positioned at $60 \mathrm{~m}$ from the release (Fig. 9 only shows the sensors at an elevation of $1.4 \mathrm{~m}$ ). This is likely due to different positioning of the sensors relative to the pipes in the congested region (the same effect was also observed in a test with a similar layout). As the focus in the present work is mainly on the quasi-steady concentrations at ignition time, the time of arrival of the flammable cloud is not considered further here.

At the time of ignition in test 8 , Fig. 7 - Fig. 9 show that the measured period maximum concentrations in at least $3 / 4$ of the rig length are between $10-11.5$ vol\% natural gas in air. The concentrations are increasing slightly along the centreline up to $62.5 \mathrm{~m}$ from the point of release. At the rear end of the rig, in the wake of the jet, concentrations are lower (around 7 vol\%). The jet velocity decreases 
significantly inside the congestion - measurements from the turbulence probes indicate that the mean flow velocity is less than $10 \mathrm{~m} / \mathrm{s}$ at $60 \mathrm{~m}$ from the release (i.e. halfway through the congested region). The velocity was likely further reduced in the region from $60 \mathrm{~m}$ to $65 \mathrm{~m}$. Due to low flow velocities, changes in gas concentration require a longer time to propagate through the congestion compared to the "free jet" conditions.

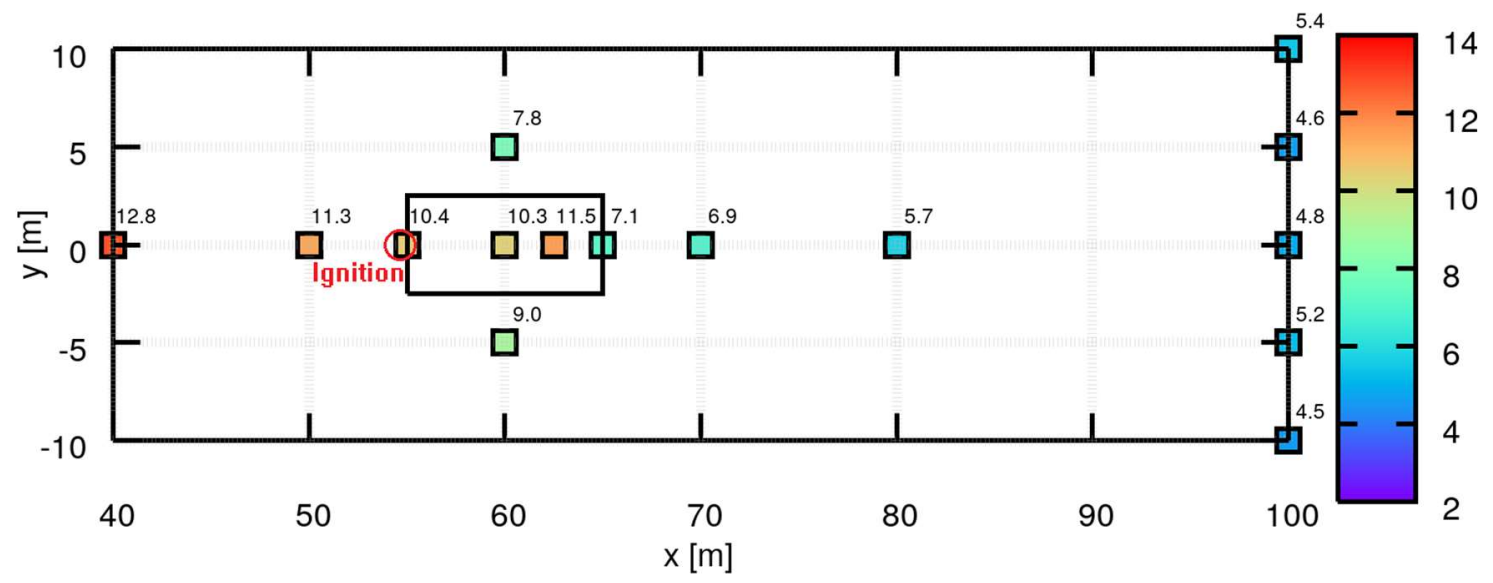

Fig. 7. Measured mole fractions $1.4 \mathrm{~m}$ from ground level in test 8 , period maximum over $5 \mathrm{~s}$ before ignition. Here, $x$ and $y$ are zero at the release point. The red circle marks the ignition location.

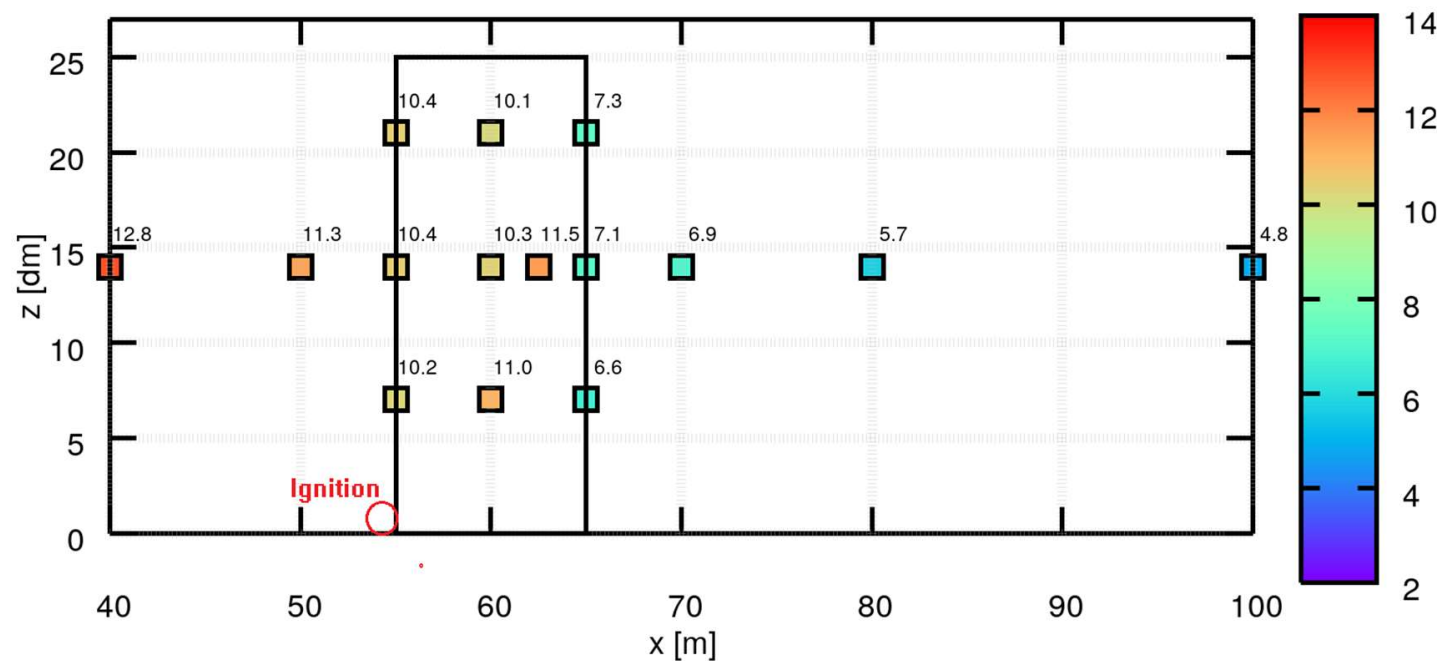

Fig. 8. Measured mole fractions at release centre line $(y=0)$ at different elevations from ground level in test 8 , period maximum over $5 \mathrm{~s}$ before ignition. Here, $x$ and $y$ are zero at the release point. The red circle marks the ignition position.

Fig. 7 indicates that the fuel concentration is near uniform across the jet $100 \mathrm{~m}$ downstream of the release point, with slightly higher concentrations on the positive side of the y-axis. Since flow speeds in the jet are low here, this asymmetry may be caused by the ambient wind.

Fig. 10 shows the measured concentrations (maximum over $5 \mathrm{~s}$ period before ignition) at the release centreline at different elevations from ground level in the "free jet" test 15 (it is important to note that the location of sensors differ somewhat between test 8 and test 15). In test 15, the concentrations appear to uniformly decrease with increasing distance from the point of release. Mass release rates and atmospheric conditions were similar for test 15 and test 8 , however, the measured concentrations upstream of $60 \mathrm{~m}$ were somewhat higher in test 8 than in test 15. 


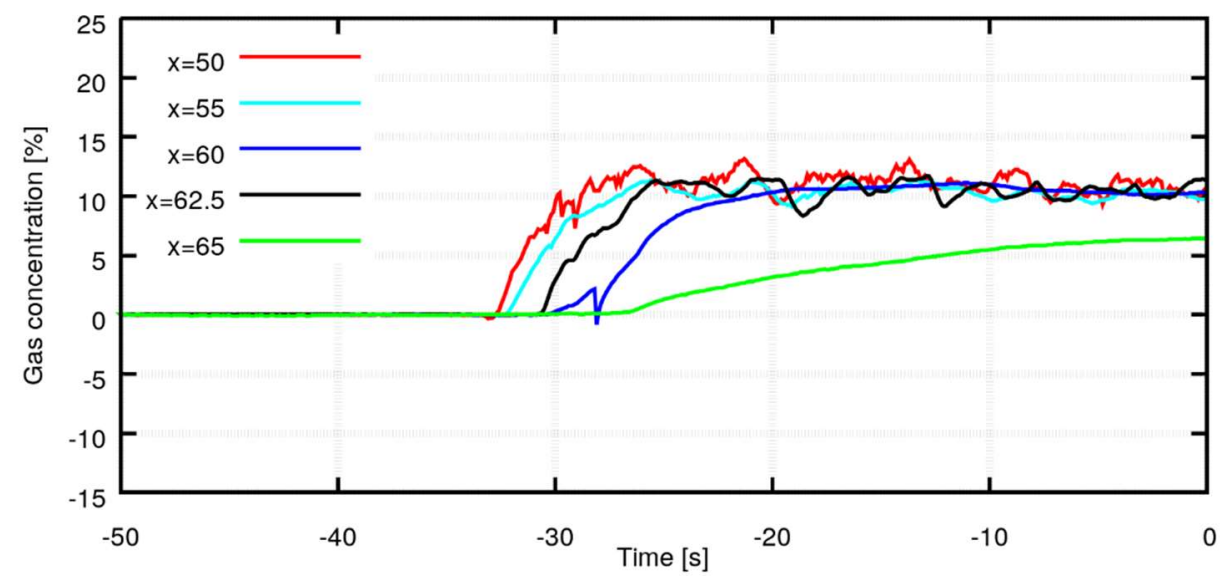

Fig. 9. Measured mole fractions at release centre line $(y=0)$ at different distances from the release point in test 8 . The sensors are placed $1.4 \mathrm{~m}$ from the ground. Ignition occurred at $0 \mathrm{~s}$.

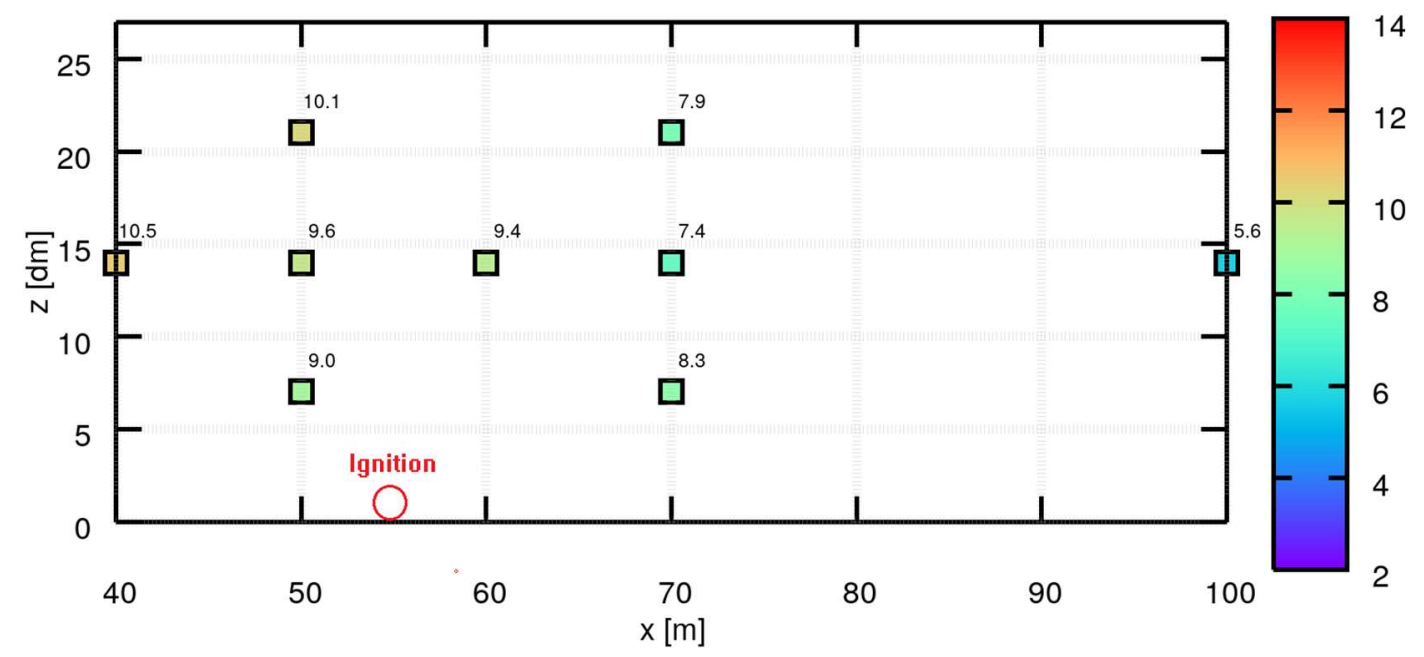

Fig. 10. Measured mole fractions at release centre line $(y=0)$ at different elevations from ground level in test 15, period maximum over $5 \mathrm{~s}$ before ignition. The red circle marks the ignition position.

Fig. 8 and Fig. 10 indicate that the period maximum concentrations (over $5 \mathrm{~s}$ before ignition) are relatively uniform in the vertical direction in the region between $55 \mathrm{~m}$ and $65 \mathrm{~m}$ from the release stratification is not observed in either test.

\subsection{Turbulence measurements}

In some of the large-scale tests in the AIRRE project, up to 20 differential pressure sensors were installed to measure turbulence in the flow. The demanding conditions under which the sensors operated during the tests, i.e. high-velocity flows with excess debris present and instrumentation masts that occasionally fell, caused several sensors to malfunction or the recorded data to be invalid. In addition, the high explosion pressures generated in several of the tests damaged many of the turbulence sensors. It was therefore necessary to replace several sensors after these tests. This effectively limited the number of tests where turbulence measurements could be performed. It was also challenging to obtain reliable recordings for a sufficiently long period to enable the extraction of turbulence velocities. Ineris performed the analysis of the turbulence measurements (Jamois et al., 2020), and retained signals from 14 pressure time series from the unobstructed tests and 50 from the tests with congested regions to evaluate the turbulence fields. Most of the measurements were taken at an elevation of $1.4 \mathrm{~m}$, while a limited number of sensors were placed either at $0.7 \mathrm{~m}$ or $2.1 \mathrm{~m}$.

The measured dynamic pressure was used to estimate the velocity in the direction of the jet axis. The integral time scale of turbulent fluctuations was obtained from the autocorrelation function, while the integral length scale was obtained by multiplying the latter by the average velocity at the same point. 
In the tests without obstructed regions, the jet mean velocity along the release direction $($ at $y=0)$ was around $70-90 \mathrm{~m} / \mathrm{s}$ at $30 \mathrm{~m}$ from the release point, $50-60 \mathrm{~m} / \mathrm{s}$ at $40 \mathrm{~m}, 40-60 \mathrm{~m} / \mathrm{s}$ at $50 \mathrm{~m}$, around 30 $\mathrm{m} / \mathrm{s}$ at $80 \mathrm{~m}$, and approximately $20 \mathrm{~m} / \mathrm{s}$ at $100 \mathrm{~m}$. Downstream of $40 \mathrm{~m}$ from the release point, the relative turbulence intensity was estimated to vary between $20-40 \%$ along the release centreline. The average turbulent velocity in the region between $40 \mathrm{~m}$ to $60 \mathrm{~m}$ was estimated to be approximately $12 \mathrm{~m} / \mathrm{s}$.

In the tests with rigs installed, the mean velocity was significantly reduced (to below $20 \mathrm{~m} / \mathrm{s}$ ) inside and just downstream of the congested region. Very few probes gave reliable data inside the rig, but the few available results indicated maximum turbulence intensities of around $40 \%$ inside the congestion. Upstream of $5 \mathrm{~m}$ from the rig, and downstream of $15 \mathrm{~m}$ from the congestion, results seemed to follow the same trend as in the tests with un-obstructed jets. The estimated values for the integral length scale for the tests without obstructed regions were (in line with the free jet theory) between $1 \mathrm{~m}$ and $2.5 \mathrm{~m}$ up to $60 \mathrm{~m}$ from the release point. Further downstream, the integral length scales were reduced to around $1 \mathrm{~m}$ or less and appeared to be approximately constant with increasing distance from the release point. Jamois et al. (2020) discuss the turbulence measurements in the AIRRE experiments in more detail.

\subsection{Pressure-time recordings and flame speeds}

Up to 20 pressure sensors were distributed throughout the region of interest in the high-pressure release experiments. During the tests, the transducers were subject to significant mechanical vibration, debris throw and heat post-ignition. Different levels of noise due to electrostatic and mechanical variations in the mounting, cabling and signal conditioning arrangement of each transducer were present, to varying degrees, in many of the pressure-time traces. Where possible, traces were corrected for these effects to leave only the relevant pressure event. To account for velocity head and temperature effects pre-ignition (from the cold jet), the traces were balanced for zero-pressure at the time of ignition.

Due to the highly turbulent, high-velocity nature of the releases, debris from the ground was entrained in the jet. Furthermore, the release produced condensed water droplets that were entrained and transported by the jet flow, significantly affecting the visibility of the region downstream of the release point, see Fig. 4 (top). Debris, water vapour and mechanical vibration can lead to a spurious trigger of the circuitry in the ionisation probes used for flame detection. These issues complicated the analysis of the ionisation probe measurements.

However, the high-speed video recordings provided useful information on the flame speeds. A tailormade, in-house computer program was used by DNV GL to evaluate the changes in pixel intensities along three lines (at different heights) with respect to time. Fig. 11 illustrates results from the video analysis performed by DNV GL at two flame positions for test 8 . The change in pixel intensity for each frame (relative to the initial frame) along the three straight lines (blue, green and red) was evaluated automatically, to produce a flame position and a corresponding time relative to the start of the analysis. This information was used to estimate a flame speed along each of these three lines, using a linear regression fit based on a user-defined set of frames over which to fit. The speed was only recorded if the linear regression showed a maximum error of $10 \%$ or less. However, as the presence of water vapour reduced the visibility of the flame in most tests, the interpretation of this data was not straightforward, as shown in Fig. 11. Where it was not possible to determine a flame speed, it was set to zero in the analysis. Flame speeds were therefore also estimated using manual definition of the flame position on each video frame (illustrated by the magenta line in Fig. 11). This manual approach was found to be more effective and is used in the flame speed estimates presented here. It should be noted that though there are significant uncertainties in measuring flame speeds from frame to frame, the overall view is still useful to consider together with the measurements of explosion pressure. 
In Fig. 11 (top), the flame had propagated around $5 \mathrm{~m}$ from the edge of the congestion, and the flame speed was still below the speed of sound in the mixture. Fig. 11 (bottom) shows the flame position at the maximum observed flame speed of $821 \mathrm{~m} / \mathrm{s}$. At this point, the flame had propagated approximately $9.3 \mathrm{~m}$ from the ignition point and had entered the fast deflagration regime (Oran et al., 2020).

Fig. 12 shows the calculated flame speed as a function of distance from the release point, estimated from high-speed video recordings of test 8 . The flame speeds in test 8 are plotted together with those from test 16 . Due to the presence of excessive water vapour in test 8 , the visibility of the flame was poor. Furthermore, the high explosion overpressures that were generated in the test lead to loss of the ionisation probe data. Therefore, significant uncertainty is associated with the flame speed estimates, in particular for the flame propagation through the first $5 \mathrm{~m}$ of the rig.

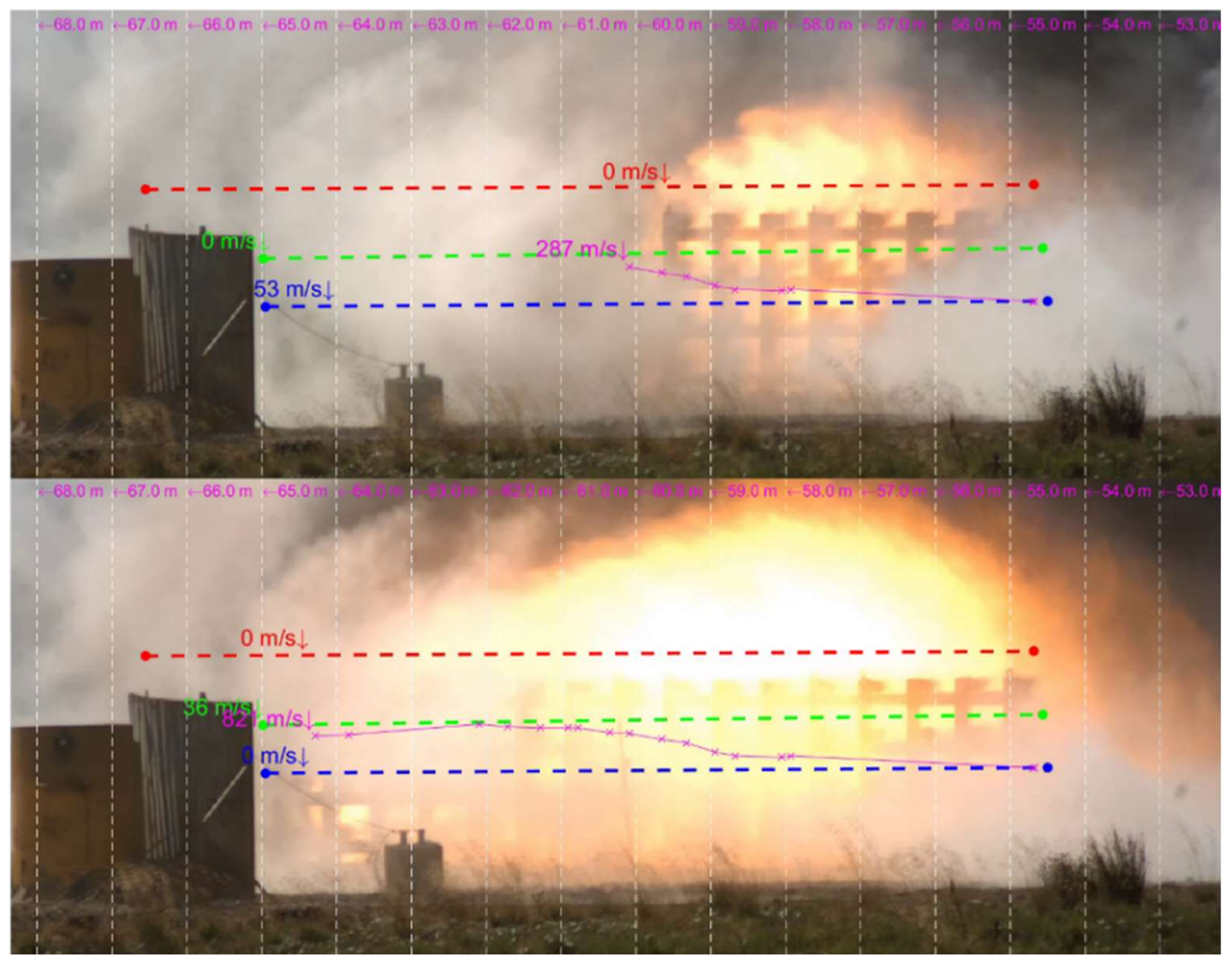

Fig. 11. Estimated speed of flame front as the flame enters the second half of the congested region (estimated to $287 \mathrm{~m} / \mathrm{s}$ at $60 \mathrm{~m}$ from the release along the manually defined flame path, marked with a magenta line) (top) and as the flame propagates towards the end of the congested region (similarly estimated to $821 \mathrm{~m} / \mathrm{s}$ at $64.3 \mathrm{~m}$ from the release, $10.7 \mathrm{~ms}$ later) (bottom) in test 8. 


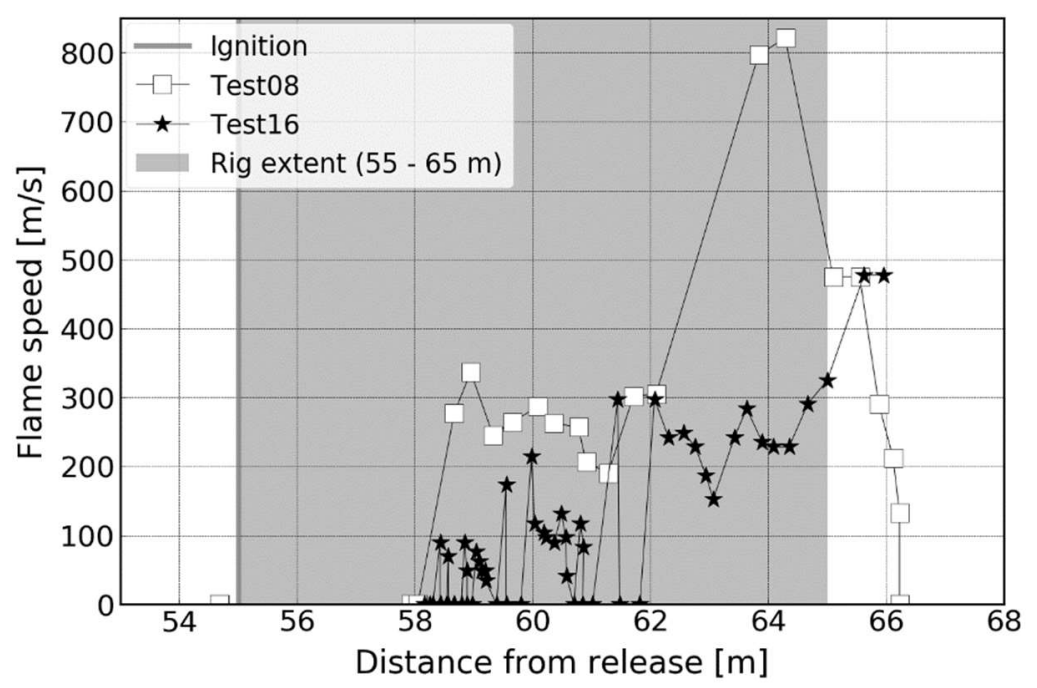

Fig. 12. Flame speed extracted from high-speed video recordings for tests 8 and 16. Test 16 is plotted for comparison with test 8 , so the rig and ignition positions are aligned. The "distance from release" is not applicable to test 16.

The measurements from the turbulence sensors in the AIRRE tests with rig 8 suggest that the relative turbulence intensities were around $40 \%$ inside the congestion, while the mean flow speeds were strongly reduced when compared to the un-obstructed cases. After the onset of the release, the flow would have generated turbulent wakes downstream of the pipes in the congested region, including turbulent shear layers that would co-exist with more energetic, coherent structures. The wakes likely interacted throughout the rig and formed a complex flow pattern prior to flame arrival. However, from the limited number of turbulence measurements (due to the challenges discussed in Section 3.3) it is not straightforward to obtain a detailed picture of the flow field internal to the rig.

In order to assess the combined effect of pre-ignition turbulence, non-zero mean flows and concentration gradients generated by the high-pressure releases, it is relevant to compare the results from test 8 to those from test 16. Fig. 13 (left) shows the peak pressure values from test 8 as a function of distance from the release point, while Fig. 13 (right) shows the pressure-time traces that were recorded inside the rig during the explosion event. A $0.1 \mathrm{~ms}$ moving average filter has been applied to the pressure signals to remove high-frequency noise.

Fig. 13 shows that the highest overpressures in test 8 were recorded at the edge of the congested region, $10 \mathrm{~m}$ downstream of the ignition point. The dominating explosion pressure-generating event in test 8 was flame acceleration through the congested region, with the highest flame acceleration occurring in the second half of the congested rig (between $60 \mathrm{~m}$ and $65 \mathrm{~m}$ from the release point).
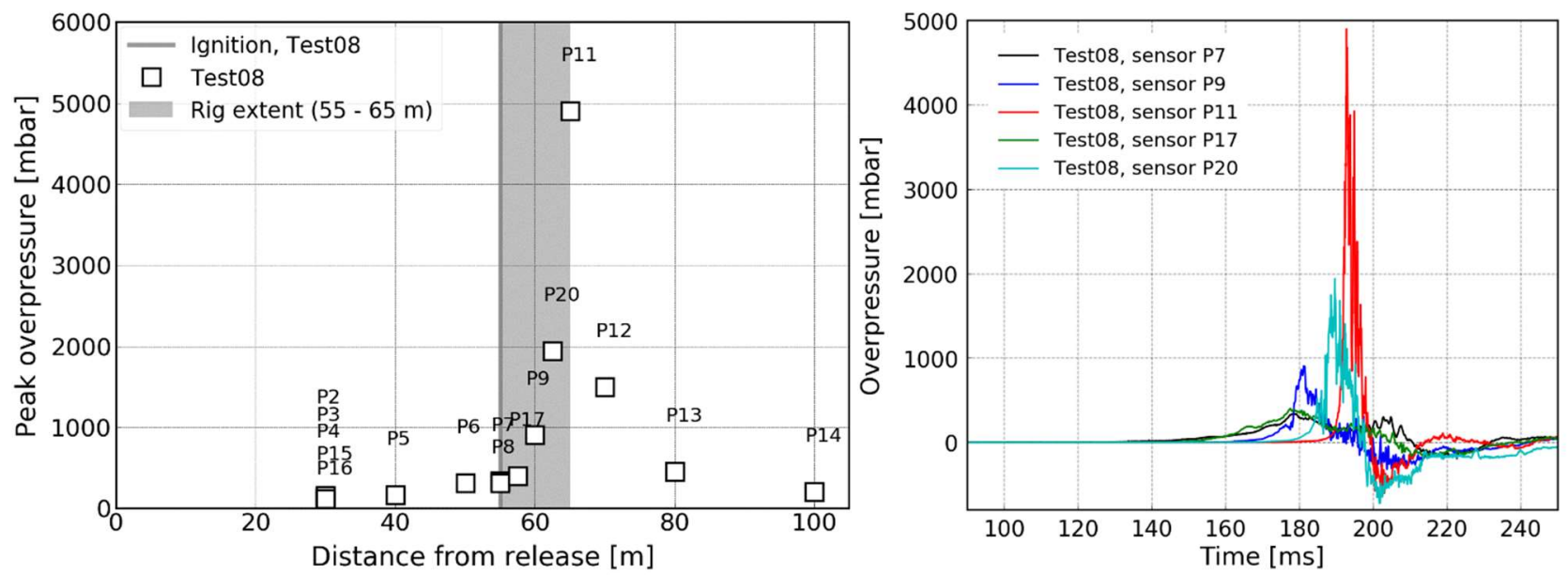

Fig. 13. Measured peak overpressures (left) and pressure-time traces from selected sensors located inside the rig in test 8 (right). 
For the flammable gas mixture used in the AIRRE experiments, the stoichiometric concentration is approximately 8.9 vol.\% natural gas in air, while the top reactivity is expected to occur around 9.7 vol.\% natural gas in air (Nilsson et al., 2019). If the concentration measurements prior to ignition are representative of the actual concentrations around the sensors at the time of flame arrival, the concentration measurements in Fig. 7, Fig. 8 and Fig. 9 suggest that the flammable cloud inside of the rig was overall fuel-rich in the upstream $3 / 4$ of the rig. Towards the downstream edge of the congested region, the concentrations were reduced quite significantly, i.e. approximately $7 \mathrm{vol} \%$. It is possible that fuel concentrations in the final $1 / 4$ of the rig, where the most significant flame acceleration occurred, were closer to the "optimal" reactivity.

Test 16 was a reference test for the experiments with high-pressure jet releases and the medium congestion level in the AIRRE project. The test was performed with a quiescent mixture with a nominally homogeneous concentration of approximately 9.2 vol.\% natural gas in air, and ignition occurred at the edge of the congestion, close to the ground (corresponding to the ignition position in test 8). Fig. 14 illustrates the position of the ignition source and the pressure transducers that were placed inside the rig in test 16, along the rig centreline. Fig. 15 (left) presents the maximum overpressures measured inside the rig, while Fig. 15 (right) shows selected pressure-time traces (corresponding to the same distance from ignition as the sensors plotted in Fig. 13 (right) for test 8).

The flame speeds in both tests are shown in Fig. 12. In test 16, reduced visibility of the flame and lack of ionisation probe data in the first $1 / 4$ of the rig lead to difficulties in extracting a reliable flame speed. However, in the first $5 \mathrm{~m}$ of the rig, the flame acceleration and generation of overpressure seem to have been modest, with regions where the flame speed was almost constant or even decelerating at times. Significant flame acceleration was observed as the flame propagated into the final $1 / 4$ of the rig, at $7-8 \mathrm{~m}$ from the ignition point. Flame acceleration seemed to persist after the flame front exited the rig, approximately $10 \mathrm{~m}$ downstream of the ignition point, as the region of highly reactive mixture extended past the rig. Despite the differences in initial conditions of the flow, the explosion event in test 16 and test 8 appears to have been qualitatively similar, cf. Fig. 12 and Fig. 15. However, in test 8 , the pre-ignition turbulence most likely enhanced the flame acceleration throughout the rig, resulting in a maximum overpressure that is approximately a factor 2.7 higher than that obtained in the quiescent reference. It is worth noting that if it is assumed that the pressure is proportional to the square of flame speed, this equates to an increase in peak flame speed by a factor of approximately 1.6, which corresponds very closely to the ratio of peak flame speeds between tests 8 and $16(800 \mathrm{~m} / \mathrm{s}$ and $500 \mathrm{~m} / \mathrm{s})$. This suggests that the estimation of peak flame speeds is relatively accurate, despite the issues already described.

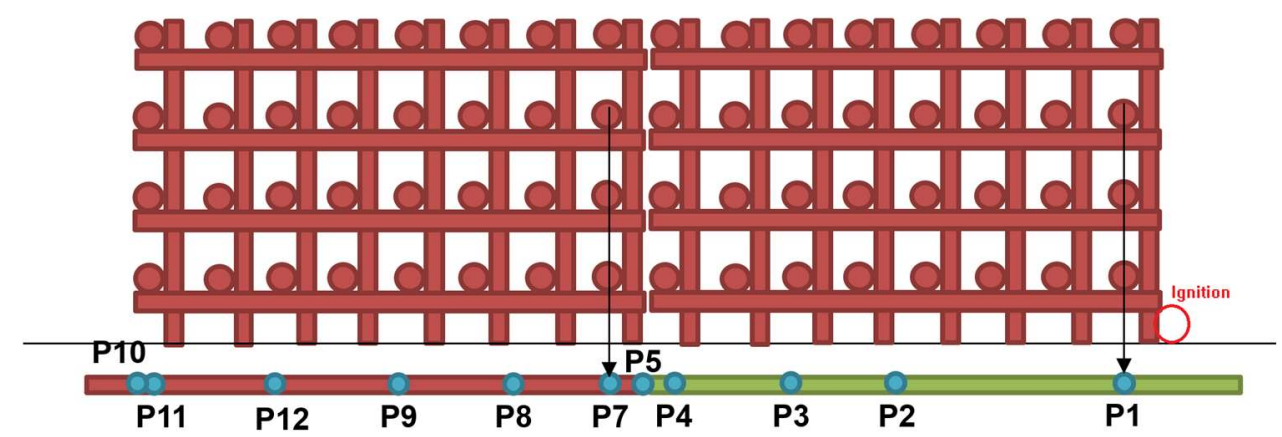

Fig. 14. Positions of pressure transducers and ignition point in test 16. 

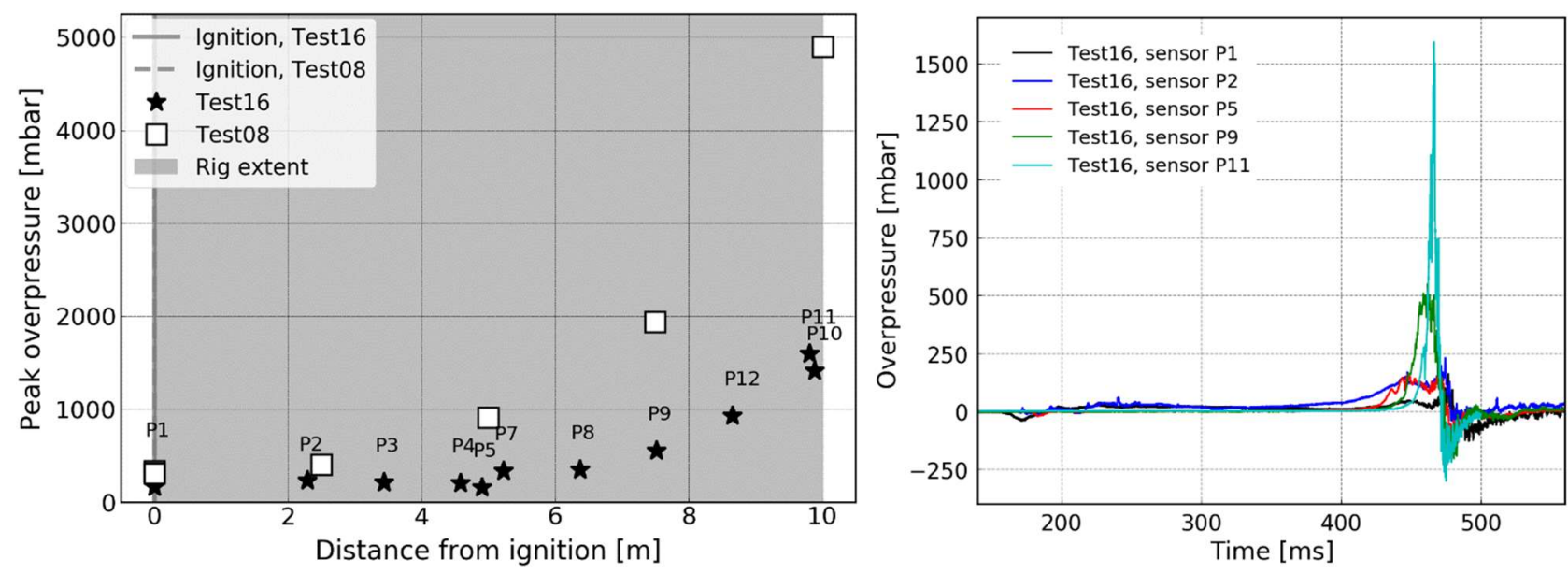

Fig. 15. Measured peak overpressures in test 16 and test 8 (left) and pressure-time traces from sensors located inside the rig in test 16 (right).

\section{Conclusions}

The primary objective for the AIRRE project was to gain improved understanding of the effect of realistic releases on the consequences of accidental gas explosions in the petroleum industry and evaluate the current state-of-the-art methodologies for quantitative risk assessment (QRA) considering the new results. The research activities in the project included a unique series of largescale explosion experiments with ignited high-momentum jet releases directed into geometries with varying levels of congestion. Selected results from these large-scale experiments are reported in this paper.

The summary of maximum overpressures from the AIRRE campaign shows that explosion severity significantly depends on the congestion level present in each test, the position of the congested region, and the ignition location relative to both the release outlet and the congested rig. The results demonstrated that an explosion propagating through a homogeneous, quiescent and nearstoichiometric (most reactive) cloud that encloses a congested region does not necessarily represent the "worst-case scenario" in terms of generated explosion overpressures. The pre-ignition turbulence generated by the high-pressure release produced higher explosions pressures than the corresponding quiescent reference test in two of the experiments with the medium congestion rig. However, in the present campaign, this only occurred when ignition happened upstream of the medium congestion rig, and the flame front accelerated with the mean flow induced by the release. In the high congestion configurations, the maximum generated overpressure seemed to be less sensitive to the position of the ignition point. However, the limited number of sensors and the variations in initial conditions complicates the analysis. Tests with corresponding configurations typically exhibited variations in period averaged concentrations of $\pm 1-2$ vol.\%, measured in the same positions. These variations will affect the reactivity of the fuel-air cloud and must be considered when results from different tests are compared.

The large-scale experiments in AIRRE also included measurements of flow velocities, turbulence levels, explosion overpressures and flame arrival times throughout the flammable gas plume. Due to the intense conditions inside the plume caused by the high-velocity jet release (as well as the high explosion pressures generated in several tests) it was challenging to obtain enough measurements to determine the properties of the flammable cloud and the ensuing flame propagation. It would therefore be beneficial to complement the results from the large-scale AIRRE campaign with smallerscale studies where more extensive measurements of turbulence, concentrations and flame speeds may be feasible, and a higher number of tests can be performed. If possible, experimental work should be combined with CFD simulations using models that are both capable of resolving part of the turbulence spectrum and represent the enhancement in flame surface area from various physical phenomena. 
Finally, the modelling work performed as part of the AIRRE project suggests that scenarios that entail flame propagation past obstacles in established, turbulent flow fields, may not be straightforward to represent with the commonly used consequence models. However, to assess whether the present results should affect the current state-of-the-art QRA methodologies requires a thorough treatment work on modelling and methodology will therefore be reported in subsequent publications.

\section{Acknowledgements}

The authors gratefully acknowledge the financial contribution to the AIRRE project from the Research Council of Norway (RCN) under grant agreement no. 269360 in the Demo 2000 program. Gexcon gratefully acknowledges the financial contributions from the sponsors GRTgaz, TOTAL and Shell Global Solutions, as well as the technical contributions from the subcontractors DNV GL Spadeadam Research and Testing and Ineris.

\section{References}

Bjerketvedt, D., Bakke, J.R. \& van Wingerden, K. (1997). Gas explosion handbook. Journal of Hazardous Materials, 52: 1-150.

CCPS (1994). Guidelines for evaluating the characteristics of vapour cloud explosions, flash fires and BLEVEs. Center for Chemical Process Safety (CCPS), New York.

City Technology. (2017). Product Datasheet AO2 Oxygen Sensor. https://www.citytech.com/engb/PDF-Datasheets/ao2.pdf. Accessed on 17 Feb. 2020.

Cronin, P. \& Wickens, M.J. (1986). A large scale experimental study of the conditions required for sustained high speed flame propagation in a flammable vapour cloud. Report No. MRS I 4348. British Gas Corporation, UK.

Davis, S., Engel, D., Groethe, M. \& van Wingerden, K. (2016). Large scale deflagration to detonation testing. Eleventh International Symposium on Hazards, Prevention and Mitigation of Industrial Explosions (11 ISHPMIE), Dalian, 24-29 July 2016, pp. 110-124.

Davis, S.G., Pagliaro, J., Botwinick, D., van Wingerden, K., Allason, D. \& Johnson, M. (2018). Don't believe the hype: using case studies and experimental evidence to show why HSE is wrong about exploding DDTs. Proceedings Fourteenth Global Congress on Process Safety (GCPS 2018), Orlando, Florida, 22-25 April 2018, 24 pp.

Hansen, O.R., Gavelli, F., Davis, S.G., Middha, P. (2013). Equivalent cloud methods used for explosion risk and consequence studies. Journal of Loss Prevention in the Process Industries, 26 : 511-527.

Harris, R.J. \& Wickens, M.J. (1989). Understanding vapour cloud explosions - an experimental study. Fifty-fifth autumn meeting of the Institution of Gas Engineers.

Hjertager, B. H. (1984). Influence of turbulence on gas explosions. Journal of Hazardous Materials, 9(3): 315-346.

ISO 5167-1:2003. (2003). Measurement of fluid flow by means of pressure differential devices inserted in circular-cross section conduits running full - Part 1: General principles and requirements.

ISO 5167-2:2003. (2003). Measurement of fluid flow by means of pressure differential devices inserted in circular cross-section conduits running full - Part 2: Orifice plates.

Jamois, D., Hébrard, J., Leprette, E., Proust, C., Mauri, L., Hisken, H., Atanga, G., Lucas, M., van Wingerden, K., Skjold, T., Quillatre, P., Dutertre, A., Marteau, T., Pekalski, A., Jenney, L., Allason, D. \& Johnson, M. (2020). Turbulence in real flammable gas releases. Thirteenth International Symposium on Hazards, Prevention and Mitigation of Industrial Explosions (13 ISHPMIE), Braunschweig, Germany, 27-31 July.

Johnson, D.M. \& Cleaver, R.P. (2002). Gas explosions in offshore modules following realistic releases (PHASE 3B): final summary report. Advantica Technology, UK. Report number: R4853. 
Johnson, D.M., Cleaver, R.P., Puttock, J.S. \& van Wingerden, C.J.M. (2002). Investigation of gas dispersion and explosions in offshore modules. Offshore Technology Conference (OTC), Houston, Texas, 6-9 May 2002, Paper OTC 14134: 7 pp.

Johnson, D.M., Tomlin, G.B. \& Walker, D.G. (2015). Detonations and vapor cloud explosions: Why it matters. Journal of Loss Prevention in the Process Industries, 36: 358-364.

Marsh (2018). The 100 largest losses 1978-2017 - Large property damage losses in the hydrocarbon industry. $25^{\text {th }}$ edition.

Moen, I.O., Donato, M., Knystautas, R., Lee, J.H. (1980). Flame acceleration due to turbulence produced by obstacles. Combustion and Flame, 39(1): 21-32.

Nilsson, J.K.E, van Sprang, A., Larfeldt, J. \& Konnov, A.A. (2019). Effect of natural gas composition on the laminar burning velocities at elevated temperatures. Fuel, 253: 904-909.

Oran, E.S., Chamberlain, G. \& Pekalski, A. (2020). Mechanisms and occurrence of detonations in vapor cloud explosions. Progress in Energy and Combustion Science, 77: 100804. DOI: https://doi.org/10.1016/j.pecs.2019.100804.

PCB Piezotronics. (2020). https://www.pcb.com/products?m=113B26. Accessed on 17 Feb. 2020.

Schelkin, K.I. (1940). Influence of tube roughness on the formation and detonation propagation in gas. Zhurnal Eksperimentalnoi i Teoreticheskoi Fiziki, 10:823-827.

Skjold, T., Hisken, H., Mauri, L., Atanga, G., Bernard, L., van Wingerden, K., Foissac, A., Quillatre, P., Blanchetière, V., Dutertre, A., Kostopoulos, D., Pekalski, A., Allason, D., Johnson, M., Jenney, L., Leprette, E. \& Jamois, D. (2018). Assessing the influence of real releases on explosions: motivation and previous work. In Proceedings of the Twelfth International Symposium on Hazards, Prevention and Mitigation of Industrial Explosions (12 ISHPMIE), Kansas City, 12-17 August 2018: 24 pp. Fike Corporation, Blue Springs, MO, USA.

Stewart, J. \& Gant, S. (2019). A review of the Q9 equivalent cloud method for explosion modelling. FABIG Newsletter, Issue 75, March 2019: 16-25.

Tomlin, G. \& Johnson, D.M. (2013). A large scale study of the venting of confined explosions into unobstructed and congested flammable vapour clouds. Proceedings Seventh International Seminar on Fire and Explosion Hazards (ISFEH), Providence, 5-10 May 2013, Research Publishing, Singapore: 679-688. ISBN: 978-981-07-5936-0. 